\title{
ID documents matching and localization with multi-hypothesis constraints
}

\author{
Guillaume Chiron, Nabil Ghanmi, and Ahmad Montaser Awal \\ Research Department \\ ARIADNEXT \\ Cesson-Sévigné, France \\ \{guillaume.chiron, nabil.ghanmi, montaser.awal\}@ ariadnext.com
}

\begin{abstract}
This paper presents an approach for spotting and accurately localizing identity documents in the wild. Contrary to blind solutions that often rely on borders and corners detection, the proposed approach requires a classification a priori along with a list of predefined models. The matching and accurate localization are performed using specific ID document features. This process is especially difficult due to the intrinsic variable nature of ID models (text fields, multi-pass printing with offset, unstable layouts, added artifacts, blinking security elements, nonrigid materials). We tackle the problem by putting different combinations of features in competition within a multi-hypothesis exploration where only the best document quadrilateral candidate is retained thanks to a custom visual similarity metric. The idea is to find, in a given context, at least one feature able to correctly crop the document. The proposed solution has been tested and has shown its benefits on both the MIDV-500 academic dataset and an industrial one supposedly more representative of a real-life application.
\end{abstract}

\section{INTRODUCTION}

Identity document capturing has become required for many online services (Bank account, online gambling, ...) that have to satisfy Know Your Customer (KYC) related regulations (Anti-Money Laundering (AML), Customer Identification Program (CIP), etc.). A registration phase allows creating the user profile based on his ID after an advanced verification is performed to ensure the document authenticity. The main question is how to fit a given input document image captured in the wild into its corresponding predefined layout (assuming it has been identified) to perform further location dependent analysis (various fields matching and decoding, security features and format checking). An automatic ID document detection and localization approach must address the following challenges:

- Handling a big number of classes (sometime close to each other, e.g. just a logo "EU" vs "UK" and few variable text field, with various formats). The number of classes for an international coverage easily reaches a thousand.

- Not well defined borders challenging traditional crop approaches: missing document edges (e.g. out of bound, occluded), borders not clearly defined (transparent, scratched, damaged), visual delimitation not corresponding to the document itself (fold marks in some paper documents, superposition of documents, mosaic prepared by a user for a scan, etc. Also, in some cases, the region of interest expected could be a sub part of the document and not delimited by obvious borders.
As explained in the next section, document localization can be achieved based on, for example, border or corner detection, local descriptors (based on keypoints), or text driven. However, ID documents high variability makes it hard to adopt a unique technique. As shown in Figure 1, one can observe how those approaches might fail for some specific document models. In addition, ID document analysis is a specific domain where deep-learning approaches are hard to build up from ground and to maintain as new models are constantly emerging. Whereas existing academic reference datasets exist and some effort has recently been provided to improve the field, they are very limited in terms of intra-class variability (for obvious confidentiality reasons). Therefore, to our knowledge, no academic dataset provides a sufficient amount of training samples (even coupled with data augmentation approaches) to set up an allin-one deep-learning model for document localization with sufficient generalisation to support a real life ever growing multi-country ID document coverage.
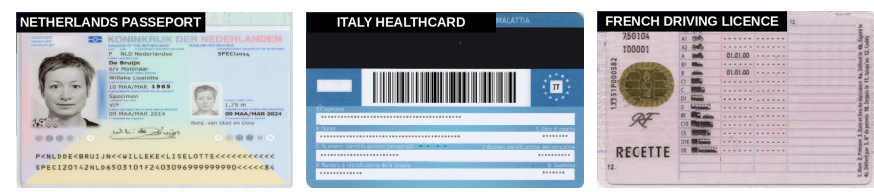

Border detection not suit- Keypoints matching not Text Driven not suitable able as borders/corners are suitable due to model due to few orthogonal text unclear (open booklets with variations (barcode, prints fields, multiple neighboring mutli pages) offsets), textureless, few field that are tight and ranachorable keypoints domly defined.

Fig. 1. Example of difficulties for specific models, illustrating the need of an hybrid approach.

To our knowledge, the ID documents classification and localization problem would gain to be more covered in the literature as it involves many various challenges. In this paper, as a minor contribution, we first make an inventory of document localization features able to spot ID documents in the wild. Then, as the main contribution, we present a novel multi-hypothesis cropping approach able to combine in complementary manner several heterogeneous features. To that end, a new original visual similarity metric is defined and used to guide the selection of the best hypothesis.

Our solution is built upon a classification approach proposed earlier in [4]. In this context, we show the importance of rejection capacities in a document classification system. Both 
classification and localization (crop) approaches are evaluated on the MIDV-500 public dataset [2] and also on a more challenging private one built from a real industrial workflow.

The rest of this paper is organized as follows. First, some related works and existing benchmarks are summarized in Section II. The proposed multi-hypothesis approach is then presented in Section III along with a list of potential usable features. Experiments and an ablation study are then detailed in Section IV, followed by the conclusion and some perspectives.

\section{STATE OF THE ART}

\section{A. Document localization approaches}

The existing document localization approaches can be divided into two main families depending on whether they use or not some a priori on the document localization: blind approaches and model-driven approaches.

Blind cropping approaches. This kind of approaches does not use any information about the searched documents. They are based on generic document features such as borders, corners, and sometimes generic content (e.g. text lines).

A content-aided approach is proposed in [22]. It consists in finding text-like areas with projection profiles along with a connected component analysis. Then a border-based method identifies a quadrilateral that seems to delimit a document. In [14], salient lines are firstly detected using Hough Transform. Then, a specific filtering based on contour regions is performed to select the document boundaries. Attivissimo et al. [3] propose an iterative algorithm to find the document borders, based on the arguable assumption that the document is visually separable form the background defined by the full scene. For corner detection based methods, deep learning techniques are widely used. In [9][28], two-step corner detection algorithms are proposed. Firstly, the corners are roughly detected using an attentional map [28]. Then, each predicted corner is refined using a corner-specific deep network.

These methods are particularly robust for documents with uniform background and perform well on existing academic datasets. However, they are not very appropriated to textured documents (like identity ones), or those captured in complex scenes that may contain lines and/or corners in their own content (or in the scene background) which can be confused with document borders and corners, leading to many false detections.

A more generic approach is proposed in [1] where an endto-end trainable $\mathrm{CNN}$ is used to locate the document and correct its distortion simultaneously. The used CNN can be trained to find the corners or also to directly predict the transformation matrix like in [24] and [26]. While [26] uses a CNN regression model and a hierarchy of Spatial Transformer Networks to directly estimate the homography between an image pair, [24] addresses irregular scene text rectification by directly rectifying the feature maps without any explicit homography estimation.
As stated in a Dropbox technical article ${ }^{1}$ in a closely related study, deep learning based approaches are black boxes and are therefore hard to debug. Therefore, they might not be the first choice for building a generic document spotting and localization solution. Moreover, end-to-end deep learning approaches require a large number of documents to be trained [18], which again is the main disadvantage in our context since we deal with identity documents hardly collectible in mass. Even if transferable pre-trained models could lower the need for training samples, it would not help much for validation and tests.

Model-driven localization approaches. Contrasting with blind approaches, the model-driven approaches are comparable to a template matching problem, with obviously the specific difficulties related to ID documents. When the model is unknown or not provided, a classification a priori among a list of predefined models is therefore required.

All-in-one classification and localization solutions are proposed in [4], [19], and [20]. In this context, supported document models are generated using a unique reference image captured in good conditions without any distortion. These models consist of the image keypoints and their local invariant features (e.g. SURF [5]). Additional annotations are added manually such as masks of variable areas and expected crop quadrilateral (which do not always fit physical/visible borders) [4]. The classification is achieved by maximizing the matching score between model keypoints and the query image ones. This yields a first coarse document localization which is refined by a specific one-to-one matching process applied toward the winner model. The matched keypoints are then fed into RANSAC to estimate a transformation matrix $H$.

A similar approach is followed by [19] and [20] but with additional features considered for the estimation of the transformation matrix $H$. While [19] simply checks on straight lines and quadrilateral topology, [20] revisits RANSAC to incorporate constraints on homography convexity and specific spatial dispersion of matched keypoints.

Even if the keypoints located on the document background layer are generally the most used, more contentrelated features are also used for ID document classification and localization. In [23], the national Vietnamese emblem specific graphic pattern is used into the process and helps for images captured by smartphones under various conditions (backgrounds, perspectives, lighting).

In another register, special text points are also adopted in [21] as a feature for localization.

\section{B. Benchmarking Datasets}

Privacy restrictions make it hard to publish real-life workflow reference datasets. The following tends to be used for comparing document classification and localization approaches.

MIDV-500 [2] is an annotated dataset composed of 15000 identity document images spread across 50 classes. For each

\footnotetext{
${ }^{1}$ https://dropbox.tech/machine-learning/fast-and-accurate-documentdetection-for-scanning
} 


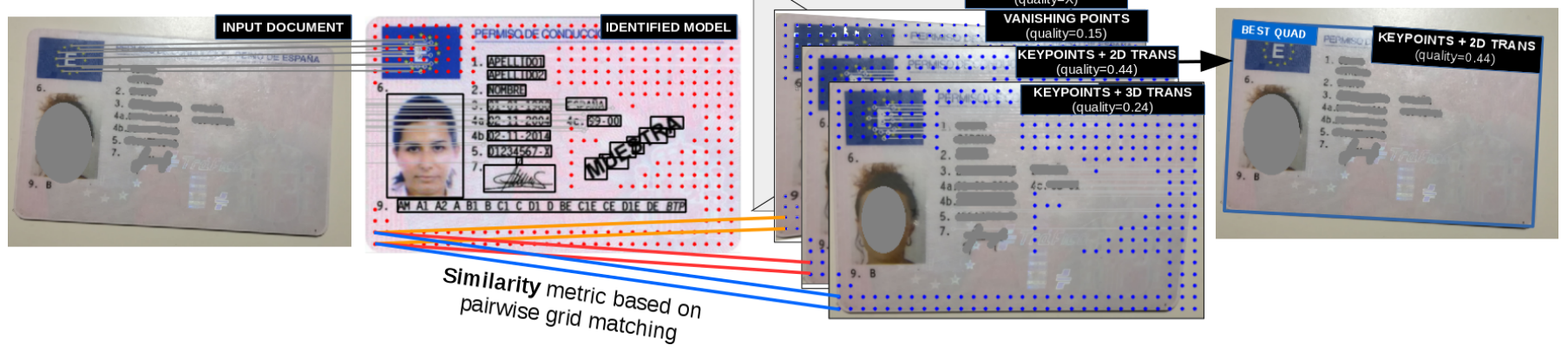

Fig. 2. Overview of the multi-hypothesis approach proposed. The best performing one was simply "KEYPOINTS $+2 \mathrm{D}$ TRANS" with a maximum similarity of 0.44 , which combines the following features: keypoints matching + RANSAC estimation with 2D affine added model constraint. The MRZ guided hypothesis could not be executed due to the absence of the required feature. The reference grid of local descriptors (e.g. SURF) used for best model selection was masked over variable areas.

class, 300 images with a resolution of $1080 \times 1920$ are captured using two different mobile devices in 5 various conditions (different backgrounds and poses). Borders and layout annotations have been performed manually for every image. Source video clips are also provided. The main drawback of this dataset is the use of only one source document per class to generate all the image samples. Also, the capturing conditions are limited as it is not real-life application data.

SmartDoc [8] dataset can also be considered relevant for document localization and correction. It consists of fully annotated video clips captured by a handheld devices. Compared to MIDV-500, it has fewer classes (not only ID one) and samples have been taken from public document databases. In total, it counts 25000 annotated frames with a resolution of $1920 \times 1080$. Whereas some attention was put at creating a challenging and representative dataset, we observe a relatively low margin of improvement among the competition results which hints the need for a more challenging datasets.

It should be noted that the two datasets are not very representative of a real workflow like the one we are dealing with in our industrial context. The latter exhibits additional challenges both in terms of document variability and image qualities. In fact, it presents a large variability in document backgrounds as well as in the region of interest itself. Moreover, industrial dataset images may suffer from various quality defects such as blur, glare, shadow, variable lighting conditions, etc.

\section{MULTI-HYPOTHESIS APPROACH}

In brief, our multi-hypothesis approach works as follows. It runs different complementary crop solutions in parallel and then selects the most competing one on the basis of a "model to query" visual similarity metric. The idea is to maximize the coverage of both model diversities and capturing conditions by automatically focusing on the most relevant features/constraints. For example, the Italian health card (see Figure 1) perfectly illustrates this need. This specific model performs poorly with a $100 \%$ keypoints matching approach [4] and would obviously benefit from a less keypoints centred solution. Figure 2 gives an overview of our approach which is composed of 3 steps:

1) Classification based on an advanced local keypoints/descriptors matching process. The main advantage of using this type of descriptors is their invariance to scale, rotation, and translation. In addition, they allow one-shot single image model construction and avoid time and resource consuming training approaches. Model construction and the classification approach is not described in this paper (as fully detailed in [4]);

2) Multi-hypothesis cropping using various features listed in the below Section III-A;

3) Best crop selection maximizing the global similarity of sparse grids of pairwise local descriptors (e.g. SURF) as described in Section III-B.

\section{A. Various exploitable features}

This section gives an overview of potential features usable to accurately localize or roughly spot ID documents in an image. Finding the quadrilateral surrounding the document is equivalent to finding the transformation matrix $H$ that fits the query image into a given model.

Figure 3 lists some features able to completely or partially solve this problem with different degrees of coverage and usability. The coverage refers to a percentage of workable document models for a given feature (estimated on the basis of a reasonably heterogeneous real-life application dataset). The usability refers to the robustness / added value the feature has by essence. None of the listed features are perfect for all the situations. The choice of these features is inspired by document intrinsic construction features (patterns, face, text, ....). Depending on the situation, some features are especially good at correcting not all but a given component of the total transformation (i.e. Orientation / Translation / Rotation / Perspective / Scale). Our bet is that combining transformations recovered by different complementary features would result in a more accurate total transformation in general. 
The percentages/marks proposed in Figure 3 are subjective estimations that helps getting an overview of the problem. For example, $95 \%$ of documents passing through our workflow seem to be localizable by the keypoints feature alone. The remaining $5 \%$ of unsupported documents, often due to lack of texture, may be handled by using complementary features as constraints while estimating the transformation. Therefore, the usability is considered high as it provides all the transformation components with a relatively high level of accuracy. On the opposite, the headpose feature alone can only be used on $30 \%$ of documents (as $70 \%$ have no face) and the usability is considered poor as face poses on photos are not as standardised (in terms of alignment) as we could expect. Also, the stateof-art headpose recovering solutions are quite unstable and inaccurate. Nevertheless, it is still a good candidate to be used as a constraint in combination with other features.

In the following, a crop hypothesis refers to complementary features used in combination. The purpose is to design different hypothesis able to produce a good crop in any situation. Their performances depend on the capturing conditions but also mainly on the document itself: texture (preferably spatially well distributed), presence of MRZ (Machine Readable Zone $)^{2} /$ distinct logos / normalized face / fixed labels, global text alignment (horizontally and vertically is better), text decoding possibilities, visible corners or/and borders, obvious detectable orthogonal lines.

\section{B. Best hypothesis selection}

To evaluate the hypothesis, we propose a visual similarity metric to be maximized to indicate the best crop for a given document model $m$. Our metric relies on an arbitrary orthogonal grid of keypoints $P_{m}$ and their corresponding descriptors $D_{m}$ extracted from the document reference image. One problem is the important variations between a given model and a query document, namely: offsets in printed layers, variable fields length, stickers, oddly distributed security elements, minor version variations. In this context, it is possible to avoid potential variable areas by exploiting eventually existing annotations already labeled in the context of the classification process [4]. Figure 2) shows such a grid with masked variable areas on the model.

During the query process, the same masked keypoints grid is then used to compute descriptors for each of the $N$ crop image candidates: $D_{c} ; c \in[1, N]$. A visual similarity metric is then computed for each candidate $c$ as follows:

$$
S_{c}=1-\operatorname{median}\left(\text { dist }_{c}\right) \text {; }
$$

where $\operatorname{dist}_{c}=\left\{\operatorname{dist}\left(D_{m}, D_{c}\right)\right\}$ is the set of all the pairwise distances between the reference (model) grid descriptors $D_{m}$ and the crop candidate descriptors $D_{c}$. Finally the winner crop $w$ is chosen by:

$$
w=\operatorname{argmax}_{c}\left(S_{c}\right)
$$

The winner crop hypothesis then provides a geometrical transformation matrix $H$ that allows the extraction and the

\footnotetext{
${ }^{2}$ https://www.icao.int/publications/Documents/9303_p3_cons_en.pdf
}

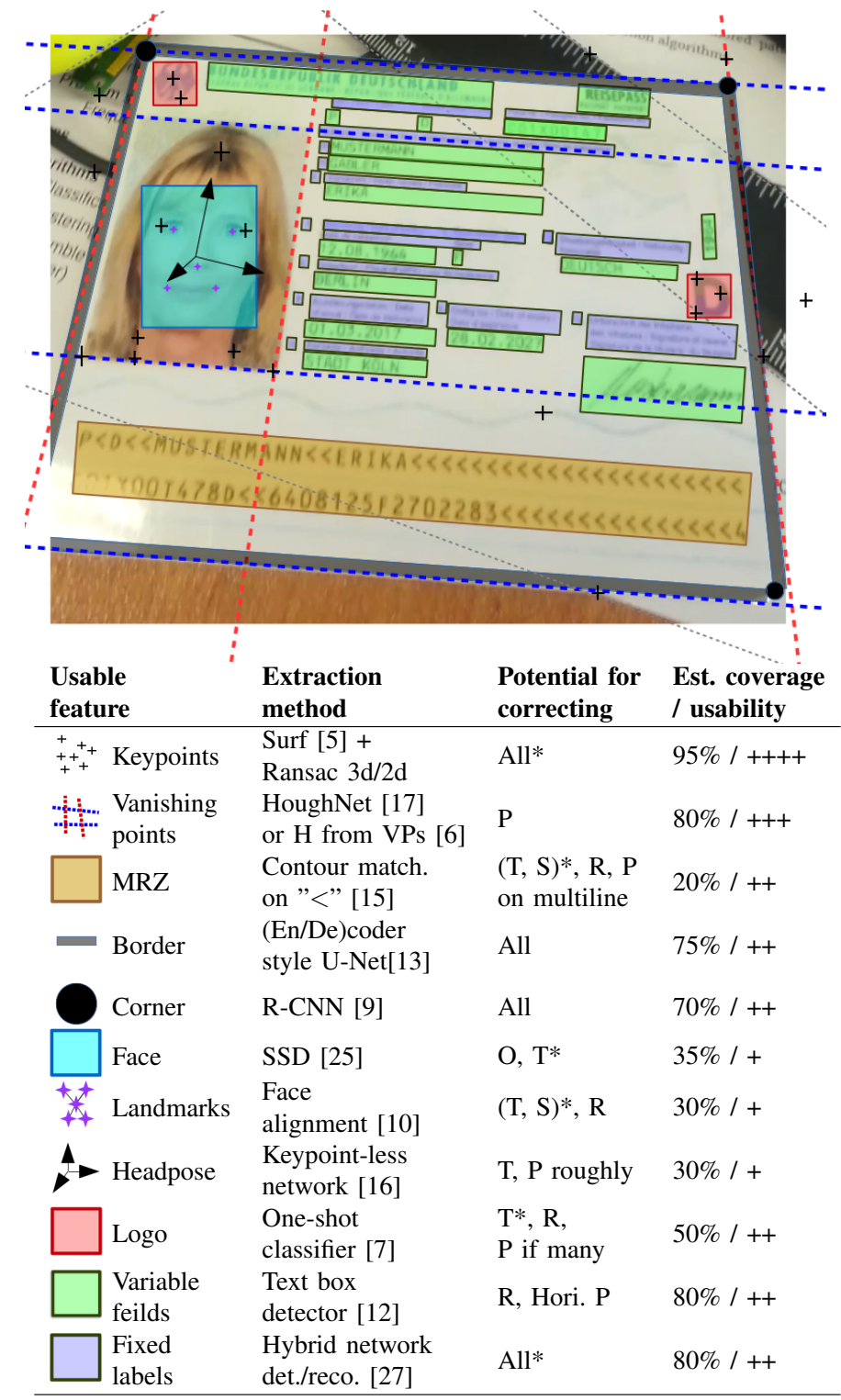

Fig. 3. Potential usable features for cropping ID documents (image source: MIDV-500 dataset). Corrections: Orientation (0, 90, 180, 270) / Translation / Rotation / Perspective / Scale. * indicates the needs of a model a priori for content matching.

correction of the document from its original image (see Figure 2).

Further quality checks are performed a posteriori to reject impossible quadrilaterals. Such a situation is usually produced when trying to crop an unknown document model, or a document-less image. In this case, further document analysis steps are impossible to apply and a rejection indicator becomes necessary to avoid false alarms or unpredictable outcomes. In this work, the geometrical quality of the winner quadrilateral is estimated as detailed in [4].

\section{IMPLEMENTATION AND EXPERIMENTS}

This section details our experiments results on a private industrial dataset as well as on the public MIDV-500 academic 
dataset. This latter provides a baseline for further work of the community.

\section{A. Datasets description}

Our industrial dataset is composed of 1587 images equitably distributed over 79 classes belonging to 14 countries and having 12 languages. Images are captured using various devices (flat scanner, handheld smartphone, triple lightning scanners) without significant quality assessment during the capture and thus very few constraints regarding external conditions (lighting, movement, etc.) or intrinsic device configurations Therefore, the document images have various dimensions, orientations, contrasts, and may be surrounded by white or complex backgrounds.

The MIDV-500 academic dataset described in Section II-B is used for the comparison.

\section{B. Four hypothesis implementation}

Not all the features presented above have been included in our experiments. Keypoints/descriptors matching stays a central aspect in all our hypothesis. Nevertheless, the incorporation of non-keypoints features compensate some of the poorly matched keypoints and thus prevent any degeneration. In this paper, we focus on four crop hypothesis (detailed below). Each of these hypothesis is a sequential combination of one or two features listed in Section III-A (Keypoints, vanishing Points, MRZ). The choice of the keypoints and vanishing points features is motivated by their large coverage and usability making them very good candidates for our first experiments. On the other hand, the use of the MRZ feature is motivated by its ability to work with no $a$ priori on the model despite a weak document coverage and an average ability to recover the perspective component. The following details the four hypothesis used in the study:

1) Keypoints $+3 D$ trans.: This commonly used approach relies on local invariant features (e.g. SURF [5]) keypoints matching and RANSAC estimation for finding the transformation matrix between the model and cropped candidate quadrilateral. The same features were more or less used in [4].

2) Keypoints + 2D trans.: Similarly to "Keypoints + 3D trans.", this hypothesis constraints the matrix to limit the transformation degree of freedom. Indeed, a considerable amount of documents (roughly estimated at more than 40\%) captured by users are almost free of any 3D perspective distortions (e.g. careful/experimented smartphone users, well designed capturing UI, scanned/pre-corrected documents). In those cases, trying to regress a transformation matrix with too many degrees of freedom leads to a lower accuracy. This hypothesis tries to compensate for this problem.

3) Vanishing Points (VP) aided perspective correction: As shown in Figure 1, models like Italian health card have obvious horizontal and vertical VPs. In this context, dominant lines are detected using Fast Line Detector [11] and are then clustered using RANSAC into dominant VPs. The most robust pairs of VPs (e.g. regarding their number of supporting lines) is then used to compute the perspective correction matrix [6]. Once the perspective component is corrected, the remaining transformation (translation + scale) is simply recovered by applying the above detailed "Keypoints + 2D trans." transformation search. The difficulty of this approach resides in the selection of a good pair of VPs supposedly corresponding to a horizontal VP and a vertical VP. In response, we propose to filter the number of detected lines at an early step by using rough assumptions regarding the raw document localization and orientation. Those early required information are estimated using again the "Keypoints + 2D trans." method which has the advantage of explicitly providing a rough $2 \mathrm{D}$ angle even on distorted images. Therefore, only the most plausible VPs pairs of VPs are kept based on spatial and rough verticality, horizontality and orthogonality criterion.

4) $M R Z$ rectangularity constraint: By standard (ISO/CEI 7501-1:2008), the Machine Readable Zone (MRZ) is a rectangular area that contains regular characters and spaces. As it is designed to be read by a machine, it appears to be relatively easy to detect (i.e. uniform background). Thus, a multi-lined MRZ can help to correct the global image perspective. To that end, the transfomation matrix is estimated by mapping the detected MRZ characters coordinates to an expected MRZ standard grid. This does not necessarily lead to accurate translation/scale results (depending on the nonnegligible XY offset of the printed MRZ). Therefore, similarly to the above "Vanishing Points" method, the remaining transformation (translation + scale) has to be recovered. The Figure4 illustrates the process.
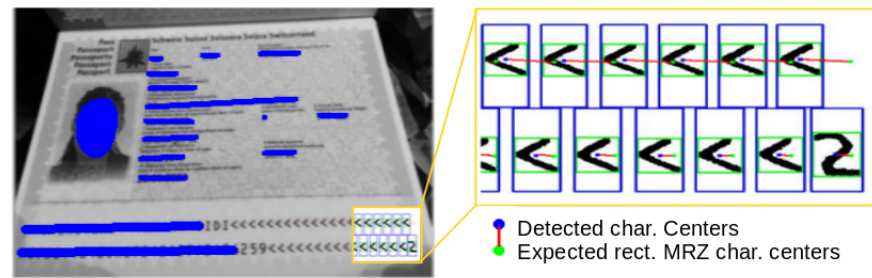

- Detected char. Centers Expected rect. MRZ char. centers

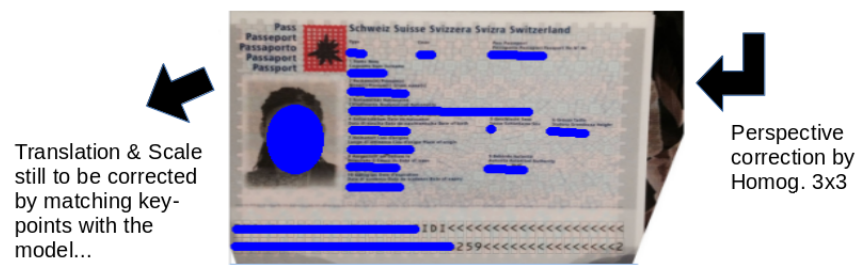

Fig. 4. MRZ rectangularity constraint illustrated. Center of the MRZ characters detected are realigned to normalized grid before application of a more constrained estimated transformation.

In the hypothesis 3 and 4 , the combination of features is applied sequentially rather than spatially. It offers an easy solution for building up, component per component, a full transformation while extracting the best from a given feature according to its robustness. Early experiments made on spatial combinations (i.e. injecting pairwise model/query MRZ characters coordinates as additional artificial matched keypoints) have shown poor results. Indeed, for a same given model of ID document, we often observe hazardous spatial offsets 
in the printed elements. Therefore, mixing various features each anchored on a distinct element (e.g. photo / MRZ / corners) often leads to an unreliable localization or unwanted distortions.

\section{Classification results}

Our approach relies on the classification method presented in [4]. As it is used as an essential early step, it is worth confirming its robustness separately. The MIDV-500 dataset contains many samples in which the document is partially or completely out of bounds and thus can not be expected to be well classified. Therefore, in the further presented experiments, we excluded samples that have out-of-bound documents by more than $10 \%$ of their area.

The left column (No rejection) of Figure 5 illustrates the classification confusion matrix on both document datasets. We can note from this column that all documents are correctly classified with an accuracy higher than $90 \%$ and many of them with an accuracy $100 \%$. It worth mentioning that one of the MIDV-500 classes is highly textured and though tends to easily absorb other classes leading to a lot of errors (last column in the lower-left confusion matrix).

The right column of this figure shows how the use of a rejection criteria (as detailed in [4]) could improve classification precision. by only rejecting miss-classified documents. The rejection feature is based on a metric able to filter most of the prediction errors and thus ensures classified samples to be good ones. On our real dataset, rejection reaches $6.8 \%$ mainly reducing errors and improving accuracy of remaining documents. With a $2.8 \%$ rejection on MIDV-500 dataset, all remaining samples are correctly classified.

\section{Localization results}

Figure 6 compares the ratio of accepted crops relative to the Jaccard index threshold. Two points of view are given: "Experimented" refers to the end-to-end effective results of our approach with automatic best hypothesis selection; "Max" shortcuts the hypothesis selection step and takes by default the best one regarding the ground truth, namely by minimizing the Jaccard index. That way, the area between the two curves shows the potential of improvement of our hypothesis selection method, while the "Max" curve shows the global potential of improvement of the hypothesis on their own.

We observe a significant difference between both datasets (academic MIDV-500 and our real). The MIDV-500 dataset shows no room for improvement regarding the selection method (both red curves are overlapped) and shows a better global hypothesis quality than our real dataset, especially for higher Jaccard index thresholds. That can be explained by 1) a difference of difficulty regarding the task or 2) a variation of the annotation quality. For the following ablation study in Section IV-E, we considered a crop quadrilateral as valid if the Jaccard index (considering to the manually annotated quadrilateral) exceeds 0.9 . We noticed that, above this value, the obtained crop is sufficient to successfully achieve further processing down the pipeline. Figure 6 shows

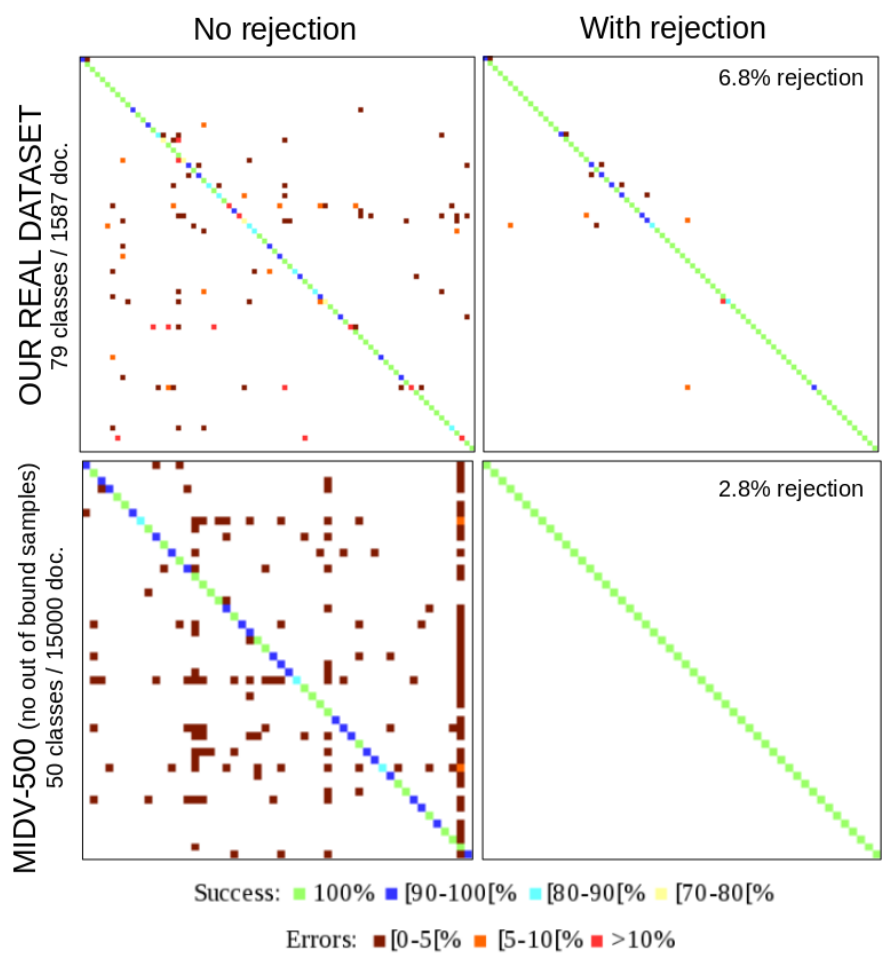

Fig. 5. Compared confusion matrices between MIVD-500 and our real dataset, with and without rejection.

that for a threshold value of 0.9 , we obtain $92.8 \%$ and $97 \%$ accepted crops on our real dataset and MIDV-500 respectively. Moreover, an improvement margin of $5 \%$ would theoretically be achievable on our dataset if we had a flawless hypothesis selection process.

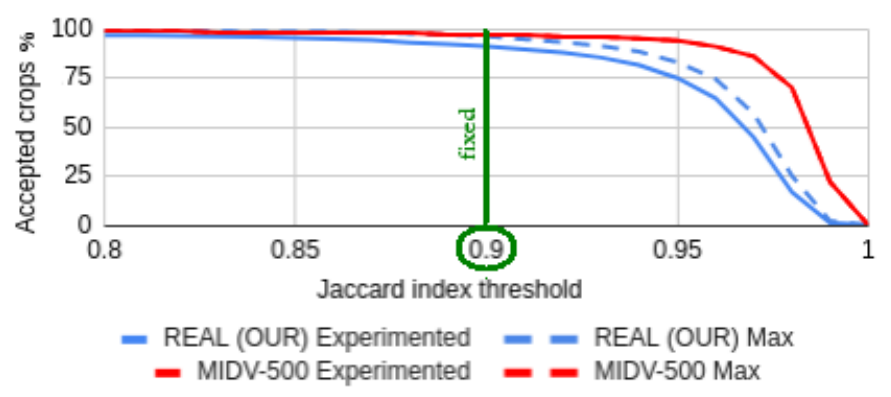

Fig. 6. Compared accepted crop ratios relatively to the Jaccard index threshold fixed between MIVD-500 and our real dataset. MIDV-500 "Experimented" and "Max" are overlapped. 0.9 is the Jaccard index threshold fixed in this study.

\section{E. Ablation study}

Among the 4 hypotheses presented in Section IV-B, different combinations have been tested. Table I shows the ability of our approach to mix different hypothesis and pick the best to achieve better results. Taken individually, no hypothesis is able to overpass an accepted crop rate of $89.3 \%$. However, when combined together, the features achieve $92.8 \%$ as an accepted crop rate. 


\begin{tabular}{|c|c|c|c|c|c|c|}
\hline \multicolumn{5}{|c|}{$\begin{array}{c}\text { Best selected hypothesis repatriation in docs \% } \\
\text { (- are ablated hypothesis) }\end{array}$} & \multicolumn{2}{|c|}{$\begin{array}{l}\text { Accepted crops } \\
>0.9 \text { Jaccard dist }\end{array}$} \\
\hline $\begin{array}{l}\text { Keypoints } \\
\text { 3D trans. }\end{array}$ & $\begin{array}{l}\text { Keypoints } \\
\text { 2D trans. }\end{array}$ & $\begin{array}{l}\text { MRZ } \\
\text { rect. }\end{array}$ & $\begin{array}{l}\text { Vanish. } \\
\text { points }\end{array}$ & $\begin{array}{l}\text { Rejected } \\
\text { (no crop) }\end{array}$ & $\begin{array}{c}\text { Detected } \\
\text { crop }\end{array}$ & $\begin{array}{l}\text { Potential } \\
\quad \max \end{array}$ \\
\hline $45 \%$ & $23 \%$ & $5 \%$ & $27 \%$ & $0 \%$ & $92.8 \%$ & $96.2 \%$ \\
\hline $61 \%$ & $33 \%$ & $6 \%$ & - & $0 \%$ & $92.5 \%$ & $94.4 \%$ \\
\hline $65 \%$ & $35 \%$ & - & - & $0 \%$ & $91.8 \%$ & $93.5 \%$ \\
\hline $48 \%$ & $24 \%$ & - & $28 \%$ & $0 \%$ & $92.2 \%$ & $95.6 \%$ \\
\hline- & $90 \%$ & $10 \%$ & - & $0 \%$ & $90.2 \%$ & $90.5 \%$ \\
\hline $100 \%$ & - & - & - & $0 \%$ & $87.8 \%$ & $87.8 \%$ \\
\hline- & $100 \%$ & - & - & $0 \%$ & $89.3 \%$ & $89.3 \%$ \\
\hline- & - & $20 \%$ & - & $80 \%$ & $18.7 \%$ & $18.7 \%$ \\
\hline- & - & - & $90 \%$ & $10 \%$ & $73.2 \%$ & $73.2 \%$ \\
\hline
\end{tabular}

TABLE I

ABLATION STUDY ON OUR REAL DATASET.

The "Best selected hypothesis repatriations" indicates the proportion of documents selected (i.e. having highest visual similarity) for each hypothesis. In a minority of cases, it happens that no hypothesis could provide a quadrilateral candidate and is therefore considered as "Rejected (no crop)", for example due to: no MRZ; no sufficient line detected to recover 2 vanishing points; geometrically absurd quadrilateral estimated by RANSAC. Indeed, the number of documents having an MRZ is about $20 \%$ in our dataset and whereas the MRZ detection process appears to be quite robust, the scope of the "MRZ rectangularity" hypothesis is finally relatively low. Let us note that documents captured with perspective distortion and flat scans-like do not necessarily fall into "Keypoints 3D trans" and "Keypoints 2D trans" respectively, but expectably a major part.

As explained in the previous section, the "Potential max" column indicates the maximum score obtainable if our feature selection method was perfect. In practice, the score can not reach $100 \%$ due to the approximation led by the crop labeling process and the variability of document formats (e.g. unclear document borders as mentioned earlier).

\section{CONCLUSION}

This paper presents, as a main contribution, 1) a novel approach for accurately localizing identity like documents. The proposed approach tests different crop hypotheses and selects the best one thanks to a custom visual similarity metric. Experiments have been run on both MIDV-500 academic dataset as well as on a supposedly more representative private one. The results have shown the superiority of our multihypothesis solution compared to a more traditional monohypothesis one.

Additionally, we note the two following side contributions: 2a) We detailed 4 crop hypothesis (or combinations of features) that perform well together, namely involving vanishing points and MRZ rectangularity constraints. $2 b$ ) We reproduced with success results of a keypoints based classification approach like [4] on both an academic dataset of reference (MIDV-500) and on a custom dataset of our own. By this occasion, we have shown the need for more challenging ID documents datasets for the community.

Following to the positive results observed in this study, we implemented the proposed solution in our industrial processing pipeline and effectively observed a posteriori a more than $1 \%$ decoding accuracy increase on several fields in our ID documents workflow (i.e. per document average on 50000 documents involving few hundreds classes), meanwhile increasing the processing time by an estimated 5\%. Although being indirect, this latter metric confirms the benefit of our proposed approach.

This study opens few interesting perspectives. For example, it could be interesting to study the impact of the grid definition for the visual similarity metric rather than using an empirically defined cell size. Obviously, it would be interesting to implement other complementary features (see Section III-A) and observe the ability of our visual similarity metric to still select the best one. As well as we use vanishing points for pre-correcting perspective, we think that using detected text boxes should help on significant number of models. We also would like to bet on the continuously improving one-shot classifiers approaches for detecting logos or distinct patterns of documents. This would completely be compatible with our non deeplearning based approach. Decoding text fields without any a priori on the layout appears to be hazardous in many cases and does not fell like a priority for identity like documents.

End-to-end deeplearning approaches are still hard to conceive today as the variety of models in circulation is constantly increasing and thus makes it hard to build from ground a generic model, even if we had access to thousands of annotated samples for many existing classes. However, works related to one-shot-classifers or siamese-like networks could become serious candidates in the future.

\section{REFERENCES}

[1] Syed Ammar Abbas and Sibt ul Hussain. Recovering homography from camera captured documents using convolutional neural networks. arXiv preprint:1709.03524, 2017.

[2] Vladimir Arlazarov, Konstantin Bulatov, Timofey Chernov, and Vladimir Arlazarov. Midv-500: A dataset for identity documents analysis and recognition on mobile devices in video stream. arXiv preprint:1807.05786, 07 2018.

[3] F. Attivissimo, Nicola Giaquinto, Marco Scarpetta, and M. Spadavecchia. An automatic reader of identity documents. In IEEE International Conference on Systems, Man and Cybernetics, pages 3525-3530, 102019.

[4] A. M. Awal, N. Ghanmi, R. Sicre, and T. Furon. Complex document classification and localization application on identity document images. In 14th IAPR International Conference on Document Analysis and Recognition, volume 01, pages 426-431, 2017.

[5] Herbert Bay, Tinne Tuytelaars, and Luc Van Gool. Surf: Speeded up robust features. In European conference on computer vision, pages 404-417. Springer, 2006. 
[6] Jean-Charles Bazin and Marc Pollefeys. 3-line ransac for orthogonal vanishing point detection. In IEEE/RSJ International Conference on Intelligent Robots and Systems, pages 4282-4287, 2012.

[7] Ayan Kumar Bhunia, Ankan Kumar Bhunia, Shuvozit Ghose, Abhirup Das, Partha Pratim Roy, and Umapada Pal. A deep one-shot network for query-based logo retrieval. Pattern Recognition, 96:106965, 2019.

[8] Jean-Christophe Burie, Joseph Chazalon, Mickaël Coustaty, Sébastien Eskenazi, Muhammad Muzzamil Luqman, Maroua Mehri, Nibal Nayef, Jean-Marc Ogier, Sophea Prum, and Marçal Rusiñol. Icdar2015 competition on smartphone document capture and ocr (smartdoc). In 2015 13th International Conference on Document Analysis and Recognition, pages 1161-1165. IEEE, 2015.

[9] Khurram Javed and Faisal Shafait. Real-time document localization in natural images by recursive application of a cnn. In 14th IAPR International Conference on Document Analysis and Recognition, pages 105-110. IEEE, 2017.

[10] Vahid Kazemi and Josephine Sullivan. One millisecond face alignment with an ensemble of regression trees. In Proceedings of the IEEE conference on computer vision and pattern recognition, pages 1867-1874, 2014.

[11] Jin Han Lee, Sehyung Lee, Guoxuan Zhang, Jongwoo Lim, Wan Kyun Chung, and Il Hong Suh. Outdoor place recognition in urban environments using straight lines. In IEEE International Conference on Robotics and Automation (ICRA), pages 5550-5557, 2014.

[12] Minghui Liao, Baoguang Shi, Xiang Bai, Xinggang Wang, and Wenyu Liu. Textboxes: A fast text detector with a single deep neural network. In Thirty-First AAAI Conference on Artificial Intelligence, 2017.

[13] Jonathan Long, Evan Shelhamer, and Trevor Darrell. Fully convolutional networks for semantic segmentation. In Proceedings of the IEEE conference on computer vision and pattern recognition, pages 3431-3440, 2015.

[14] Élodie Puybareau and Thierry Géraud. Real-time document detection in smartphone videos. In 25th IEEE International Conference on Image Processing, pages 1498-1502, 2018.

[15] Varga Robert, Costea Arthur, Szakats Istvan, and Nedevschi Sergiu. Efficient real-time contour matching. In IEEE 8th International Conference on Intelligent Computer Communication and Processing, pages 193-199, 2012.

[16] Nataniel Ruiz, Eunji Chong, and James M Rehg. Finegrained head pose estimation without keypoints. In Proceedings of the IEEE conference on computer vision and pattern recognition workshops, pages 2074-2083, 2018.

[17] A. Sheshkus, A. Ingacheva, V. Arlazarov, and D. Nikolaev. Houghnet: Neural network architecture for vanishing points detection. In International Conference on Document Analysis and Recognition, pages 844-849, 2019.
[18] Ronan Sicre, Ahmad Montaser Awal, and Teddy Furon. Identity documents classification as an image classification problem. In International Conference on Image Analysis and Processing, pages 602-613. Springer, 2017.

[19] Natalya Skoryukina, Vladimir Arlazarov, and Dmitry Nikolaev. Fast method of id documents location and type identification for mobile and server application. In International Conference on Document Analysis and Recognition, pages 850-857. IEEE, 2019.

[20] Natalya Skoryukina, Igor Faradjev, Konstantin Bulatov, and Vladimir V Arlazarov. Impact of geometrical restrictions in ransac sampling on the id document classification. In Twelfth International Conference on Machine Vision, volume 11433, pages 35-41. International Society for Optics and Photonics, 2020.

[21] Oleg A Slavin. Using special text points in the recognition of documents. In Cyber-Physical Systems: Advances in Design \& Modelling, pages 43-53. Springer, 2020.

[22] N Stamatopoulos, B Gatos, and A Kesidis. Automatic borders detection of camera document images. In 2nd International Workshop on Camera-Based Document Analysis and Recognition, pages 71-78, 2007.

[23] H. T. Viet, Q. Hieu Dang, and T. A. Vu. A robust end-toend information extraction system for vietnamese identity cards. In 6th NAFOSTED Conference on Information and Computer Science, pages 483-488, 2019.

[24] Mingkun Yang, Yushuo Guan, Minghui Liao, Xin He, Kaigui Bian, Song Bai, Cong Yao, and Xiang Bai. Symmetry-constrained rectification network for scene text recognition. IEEE/CVF International Conference on Computer Vision, 2019.

[25] Shifeng Zhang, Xiangyu Zhu, Zhen Lei, Hailin Shi, Xiaobo Wang, and Stan Z Li. S3fd: Single shot scaleinvariant face detector. In Proceedings of the IEEE International Conference on Computer Vision, pages 192-201, 2017.

[26] Q. Zhou and X. Li. Stn-homography: estimate homography parameters directly. arXiv preprint:1906.02539, 2019.

[27] Xinyu Zhou, Cong Yao, He Wen, Yuzhi Wang, Shuchang Zhou, Weiran He, and Jiajun Liang. East: an efficient and accurate scene text detector. In Proceedings of the IEEE conference on Computer Vision and Pattern Recognition, pages 5551-5560, 2017.

[28] Anna Zhu, Chen Zhang, Zhi Li, and Shengwu Xiong. Coarse-to-fine document localization in natural scene image with regional attention and recursive corner refinement. International Journal on Document Analysis and Recognition (IJDAR), 22, 082019. 